\title{
Galvanic Skin Response Data Classification for Emotion Detection
}

\author{
Djoko Budiyanto Setyohadi ${ }^{1}$, Sri Kusrohmaniah ${ }^{2}$, Sebastian Bagya Gunawan ${ }^{3}$, Pranowo $^{4}$, \\ Anton Satria Prabuwono ${ }^{5}$ \\ ${ }^{1,3,4}$ Departement of Informatics Engineering. Universitas Atma Jaya Yogyakarta, Yogyakarta 55281, Indonesia \\ ${ }^{2}$ Psychology Department, Universitas Gajah Mada, Yogyakarta, Indonesia \\ ${ }^{5}$ Faculty of Computing and Information Technology, King Abdulaziz University, Rabigh 21911, Saudi Arabia
}

\section{Article Info}

Article history:

Received Feb 12, 2018

Revised May 25, 2018

Accepted Jul 2, 2018

\section{Keyword:}

Classification

Emotion detection

Experimental research

Galvanic skin response

Support vector machine

\begin{abstract}
Emotion detection is a very exhausting job and needs a complicated process; moreover, these processes also require the proper data training and appropriate algorithm. The process involves the experimental research in psychological experiment and classification methods. This paper describes a method on detection emotion using Galvanic Skin Response (GSR) data. We used the Positive and Negative Affect Schedule (PANAS) method to get a good data training. Furthermore, Support Vector Machine and a correct preprocessing are performed to classify the GSR data. To validate the proposed approach, Receiver Operating Characteristic (ROC) curve, and accuracy measurement are used. Our method shows that the accuracy is about $75.65 \%$ while ROC is about 0.8019 . It means that the emotion detection can be done satisfactorily and well performed.
\end{abstract}

Copyright $@ 2018$ Institute of Advanced Engineering and Science. All rights reserved.

\section{Corresponding Author:}

Djoko Budiyanto Setyohadi,

Magister Teknik Informatika,

Universitas Atma Jaya Yogyakarta,

J1. Babarsari 43 Yogyakarta 55281, Indonesia.

Email: djoko.bdy@gmail.com

\section{INTRODUCTION}

Currently, human emotion is an important thing and has become a concern in various fields of research, including computer science [1]. This phenomenon is real, considering that emotion as a human expression implies activities done by human beings and their excitement can be detected based on the data measured from them: voice, face, body, and physiology [2], [3]. Detection of human emotion is potential to render the development of diverse aspects of life such as entertainment, learning, and other elements [2], [4]-[6]. The detection is part of a data mining process, namely classification, and clustering. Until today, there have been at least ten machine learning algorithms which can be used for both classification and clustering [7]. The following methods are examples of the detection algorithm based on classification and clustering, which means classifying like Support Vector Machine (SVM), Naïve Bayes, K-Nearest Neighbors $(\mathrm{KNN})$, Decision Tree and many others. They reported that the performance of classification could be enhanced when they customize the parameter within the algorithm to gain a maximum result [7].

SVM is one of the popular algorithms due to its sufficient capability in nonlinear classification, regression and outlier detection even before the algorithm became popular [7]. Although the use of SVM is more straightforward than other algorithms, to obtain an optimal result, an appropriate approach is required. Some methods that have been commonly applied include transformation, feature scaling, choosing the right kernel and finding the right $\mathrm{C}$ and $\gamma$ [8]. SVM works by detecting the hyperplane that has the most significant margin when a dimension is more than two (2), so data can be effectively separated in a linear manner (this projection is carried out in kernel technique). There are four primary kernels in SVM, namely linear, radial 
basis function (RBF), sigmoid, and poly-nominal. Among the four kernels, the RBF is worth considering to be used due to its capability in separating nonlinear data [9], [10]. With the capacity, the use of SVM can be employed as a promising alternative for emotion detection.

Emotion detection using biosensor has become popular recently. Besides its frequent use in many applications, bio censor also possesses the best performance indirectly measuring parameters in the human body, whose value is influenced by human emotion status. These sensors can measure generated-signals from human body such as heart rate, blood pressure, temperature, skin conductance, etc. Many scholars reported that the variety of measurement of generated-signals could be used as a foundation of emotion detection. [11]-[14], and one of the favorite bio censor nowadays is Galvanic Skin Response (GSR).

Galvanic Skin Response (GSR) is a tool which censors human psychological symptom. It measures signals sent by the human skin which is considered as a reflection of physiological changes [15], [16]. The use of GSR for physiological purposes is firstly implemented by Vigouroux. Moreover, many researchers reported the use of GSR for physiological phenomena [15], [17]. GSR signal indicates electrical changes measured at the surface of the human skin that varies with the changes in skin moisture level (sweating) and reflects the differences in their sympathetic nervous system [15]. The GSR signals are formed by the changing body resistance due to the changing body conductivity caused by the production of sweat. This tool has been quite favorite as it is adequately sensitive and inexpensive. In its development, the use of GSR to detect emotion can be carried out due to the spontaneous reaction which cannot be controlled by the user. Furthermore, it is considered as one of the strongest signals that can be used for emotion detection [18], [19]. Relying on the classification performance of SVM in the previous research particularly when SVM should deal with the temporal data classification, SVM was taken as the SVM algorithm to detect emotion based on GSR data.

Within this decade, a lot of researchers on emotion detection have been conducted. The algorithm of various detection tools such as GSR, electroencephalogram (EEG) and other devices have been made [3], [4], [12]. For example, EEG is used for measuring the change of positive and negative emotion of the subject who is induced through an IDFA or qDFA video. It was reported that the accuracy reached $74.3 \%$ while the accuracy of the qDFA model reached $69.4 \%$. It was also said that if it was applied to detect personal emotion, the accuracy could reach $94.5 \%$ [20]. The combination of EEG with human physiological change called Eye Movement Signals was also done [21]. They reported that when the combinations of the two factors are paired fuzzy integral fusion algorithm classification could increase the performance up to $10 \%$ so that the system accuracy became 87.59.EEG data is interesting to process and tend to have high accuracy in the context of emotion detection problems. However, EEG has several crucial issues, which are (1) expensive and (2) high noise of data resulted by its sensor. This phenomenon has made researchers attempt to find alternatives that are more appropriate, and GSR is one of the other options to choose. GSR is an alternative that has been developed since it is cheap and adequately sensitive. The two reasons at least were related to the main reason why GSR is applied to detect psychological condition using various methods. There are several studies which propose different methods of detecting stress levels by measuring skin conductance [14], [22]. An example of this research was the one conducted by Villarejo. They attempted to classify stress suffered by a person using GSR by providing stress induction in a mathematical calculation. The result was a model that was formed by using GSR data could classify stress with an accuracy that reached up to $76.56 \%$ [22].

The study described in [23] has the objective of detecting sweat levels for the diagnosis of sudomotor dysfunction, something that can help in the diagnosis of diabetes. There are other medical applications based on skin conductance, such as epilepsy control: sweaty hands may be a warning signal of an epileptic attack, as support of the diagnosis and treatment of bipolar disorder patients [24]. By combining the sweat of the hands with the temperature of the skin, it is possible to develop a truth meter [18]; as when the person is lying, his hands are colder, and skin resistance is lower. In this case, it is not necessary to include an ADC because the variation of skin resistance happens at odd times so, with different resistances and transistors, it is possible to build a lie detector. A truth meter is possibly established based on the hands sweat and skin temperature. Person's hands are colder, and he has lower skin resistance when he is telling a lie.

Attempting to do the same thing done by Villarejo, Kurniawan classified stress using GSR and noise signals [14], [22]. Classification by noise signals generated the best result, which was $92 \%$ through SVM algorithm. When the GSR data was used, however, the accuracy obtained was only $70 \%$ through the same algorithm. There was an opinion that when we attempted to classify a person's stress using another person's data, the generated accuracy would decrease [14]. Researchers commonly use GSR to classify the human stress level. In a different approach, Liu attempted to classify emotional status into happiness, grief, fear, anger, and calm using GSR data. The best accuracy level obtained by Liu was $66.67 \%$ through SVM algorithm with kernel radial based function (RBF) [12]. Recently, the Sliding Window technique has often 
been used in physiological data classification. In the research, the stress can be appropriately classified, where the accuracy of classification is up to $98 \%$ by combining HRV data with ECG data which were processed by Sliding Windows using widths 24 points, and it was classified using SVM with linear kernel [25]. A different method was carried out by Wei, who attempted to classify emotion into anger, fear, joy, sorrow, acceptance, rejection, surprise and expectancy. The research obtained a result with reasonable accuracy as much as $80 \%$ using SVM algorithm which was processed by Sliding Windows technique [12]. Using different approach, Guo also tried to classify human emotion into amusement, fear, relaxation, and sadness using Sliding Windows technique with the lag as much as 20 points. The research also obtained a good result with accuracy that reached $79.45 \%$ [18].

Support Vector Machine (SVM) was first introduced by Vladimir Vapnikin 1995 and used for binary classification purpose [26]. When SVM performs, it will create hyperplane which is aimed as a divider of each class. The basic concept was looking for the hyperplane which separated d-dimensional data correctly into two classes. The hyperplane had the biggest margin. For further explanation see Figure 1.

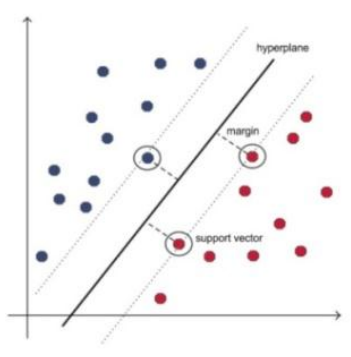

(a)

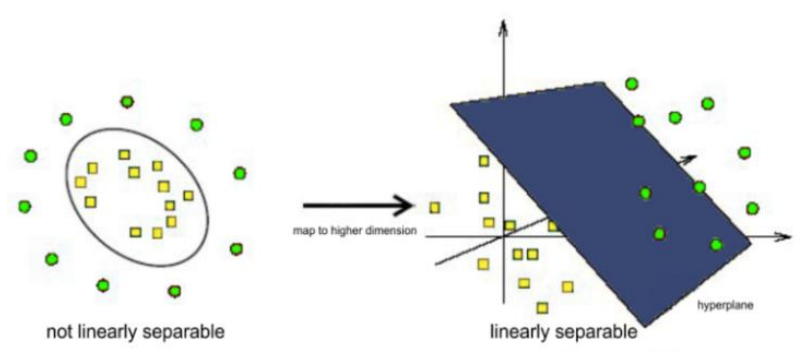

(b)

Figure 1. Support vector machine (a) and an example of kernel solving (b)

The following formula is to calculate the hyperplane in the SVM:

$$
y_{i}\left(x_{i} \cdot w+b\right) \geq 1 \quad \forall i
$$

in which $y_{i}$ is label, $x_{i}$ is input, $w$ is weight and $b$ is bias.

Although the SVM has proved to become a highly effective learning algorithm in the real world data, it has not frequently been used. It is caused by several problems found in the SVM, such as the fact that the implementation of the SVM which is not easy. Besides, the use of the wrong parameter may increase difficulty level in the computation process. Initially, the SVM was created with a method of linear problemsolving. However, there have been data that are not linear. Therefore, kernels that could be able to map the data in the dimensional space which is usually bigger (feature space) were created. Accordingly, nonlinear problems that could previously not be adequately solved could eventually be solved. There have been kernels that frequently used in SVM such as radial basis function (RBF) or Gaussian, polynomial and sigmoid. The figure shows two an example of problem-solving using kernels. However, the use of kernels has particular weaknesses. Wrong measuring parameters in kernels can cause the model to become over lift or underfit. Also, the use of kernels can increment the computation load so that it causes the process to slow down. RBF is one of the kernels, which has frequently been used since it has a good performance and tested. RBF maps nonlinear data into a bigger dimension. The hyperplane in the nonlinear data can be sought. The formula of RBF kernels data mapping is as follows:

$$
K\left(x_{a}, x_{b}\right)=\exp \left(-\frac{\|x a-x b\|^{2}}{2 \sigma^{2}}\right)
$$

in which $\sigma$ can be changed according to the needs [27]. 


\section{PROPOSED METHODOLOGY}

The research methodology of the data collection was divided into two parts. One was designed for the respondents, and the other was intended for the system. The design for the respondents used the withsubject designs, and the system design was developed by combining several methodologies that corresponded to emotional detection using audio-visual induction. In this study, as many as 39 respondents were chosen randomly from the Psychology Faculty of GadjahMada University, Yogyakarta and the Industrial Technology Faculty of Atma Jaya University, Yogyakarta, all of whom were still active students. Methodology, as an overall process, is illustrated in Figure 2. While the data collection process was done in an isolation room and the tool installation is configured in Figure 3.

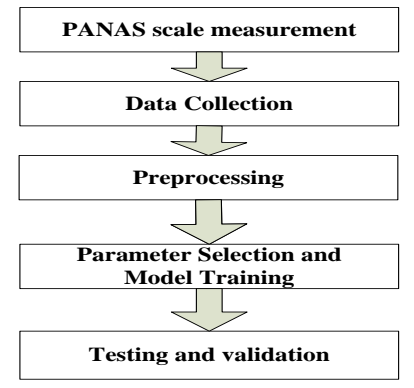

Figure 2. Research methodology

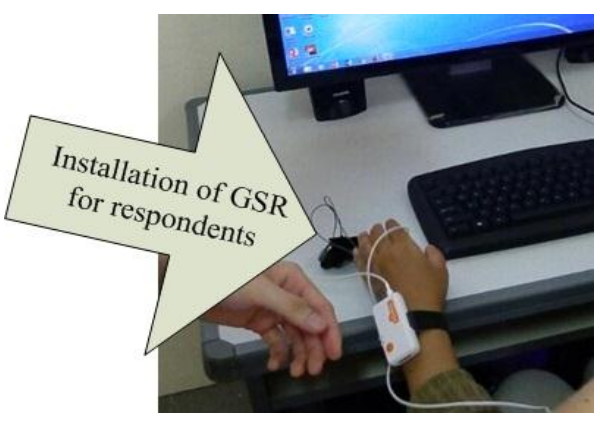

Figure 3. The GSR installation

\subsection{PANAS scale measurement}

PANAS SCALE was given at the beginning of the experiment to obtain both positive and negative affection baselines. The baseline of physiological sensor data was recorded for three minutes after the subject had finished working on the PANAS scale. During the three minutes, the respondents were required to wait for the next instruction. After three minutes, an instruction would appear on the subject to memorize unrelated pairs of words followed by the actual task given to them, which was to remember the pairs of words that had been memorized using the cued recall method. PANAS Scale is a self-report questionnaire consisting of 20 items with answers choices in the Likert scale form of 5 answer choices used to measure the level of affection [28]. We had prepared a schedule of data collection which was conducted for six days, starting from 08.30 to $16: 30$ with 30 minutes between sessions. Subject grouping in the experimental and control groups was carried out randomly, and so were the presentation of stimuli and lists of words to remember. Before data collection, each subject was required to fill in the informed consent containing the research procedures to be followed, the consequences that might occur, as well as the incentives that the subjects would receive. If they agreed, they were asked to sign the informed consent. After that, the experimenters gave an additional explanation to ensure that the subjects completely understood the experiment procedure.

\subsection{Data collection}

Data collection was done in a room which complied with the experimental protocol. The settings here included the temperature, light and noise set. The treatment of the condition and equipment was the same for all respondents, which included room, computer, screen, speakers, GSR sensor, keyboard, mouse, table, and chair.

\subsection{Pre-processing}

Pre-processing was divided into several stages, namely data categorization, data aggregation, data normalization, and data lagging. The data obtained were then categorized into three columns by the treatment given at the time of the experiment (positive, neutral, negative). This data categorization would be useful in data labeling. The next process was to aggregate the data to convert the time unit into seconds by averaging the data according to the GSR frequency at the time of data collection. In this case, the data was averaged on every 103 data. The next thing to do was normalization or feature scaling which was useful for standardizing the distance or range of variables or features in the data so that the maximum and minimum value became 1 and 0 . Finally, the data was formed by the number of $n$ lag and was added with a positive, neutral or negative label. 


$$
x=\frac{x-\min (x)}{\max (x)-\min (x)}
$$

\subsection{Parameter selection and modeling training}

In this research, the model was trained to use SVM algorithm. The number of lags that was used started from 5 to 40 lags SVM parameter tuned automatically with the cost starting from $2^{\wedge} 1$ to $2^{\wedge} 8$ using the linear kernel, polynomial, radial based function and sigmoid.

\subsection{Model testing and validation}

After the parameter required by SVM model was set, the result was validated to approve the quality of the classification model. The accuracy and Receiver Operating Curve were obtained by using the following formula:

$$
\text { Accuracy }=\frac{T P / T N}{T P+T N+F P+F N}
$$

where: TP is true positive, $\mathrm{TN}$ is true negative, and $\mathrm{FP}$ is false positive and $\mathrm{FN}$ is false negative

\section{RESULTS AND ANALYSIS}

In this section, it is explained the results of research and at the same time is given the comprehensive discussion. Results can be presented in figures, graphs, tables and others that make the reader understand easily [2], [5], [29]. The discussion can be made in several sub-chapters.

\subsection{Data set validation based on PANAS scale}

Manipulation validation was done by running Repeated Measures ANOVA on the PANAS scale. Manipulation can be declared successful if there is a different score of PANAS in a condition that is relative to the baseline and also a difference in each condition of treatment. The descriptive data of the two sub-scales of PANAS can be seen in Table 1. Sphericity validation of positive and negative affection sub scale of PANAS scale both suggested coefficient $\mathrm{p}<0.001$. Correction on sphericity was done by employing Greenhouse-Geisser (since the epsilon was above 0.7).

Table 1. Statistical Description of PANAS Scale of Positive and Negative Affections

\begin{tabular}{ccccccccc}
\hline & \multicolumn{3}{c}{ Positive Affection } & \multicolumn{4}{c}{ Negative Affection } \\
& Mean & SD & Min & Max & Mean & SD & Min & Max \\
\hline Baseline & 3.566 & 0.4828 & 2.3 & 4.3 & 2.171 & 0.6182 & 1.2 & 4 \\
Neutral Condition & 3.229 & 0.6049 & 1.5 & 4.4 & 2.021 & 0.748 & 1 & 4.1 \\
Positive Condition & 3.284 & 0.5659 & 1.8 & 4.2 & 1.992 & 0.7585 & 1 & 4.4 \\
Negative Condition & 3.239 & 0.6262 & 1.4 & 4,2 & 2.339 & 0.7387 & 1.1 & 4.1 \\
\hline
\end{tabular}

It is seen in Table 2 that the result of Repeated measures ANOVA with Greenhouse-Geisser correction resulted from coefficient $\mathrm{p}<0.001$ in the sub-scale of positive affection and $\mathrm{p}=0.002(\mathrm{p}<0.01)$ in the sub-scale of negative affect, which meant that there were significant differences between the two in at least one of the conditions. Validation that is more detailed was done by carrying out post hoc using Bonferroni method, of which result can be seen in Table 3.

\begin{tabular}{ccccc}
\hline Sub-scale & Sphericity Correction & $\varepsilon$ & $\mathrm{df}$ & $\mathrm{p}$ \\
\hline Positive Affection & Greenhouse-Geisser & 0.705 & 2.114 & $<0.001^{* *}$ \\
Negative Affection & Greenhouse-Geisser & 0.763 & 2.290 & $0.002^{* *}$ \\
\hline
\end{tabular}

Table 3. Post Hoc Bonferroni Validation of PANAS Scale; *p <0.05; **p $<0.01$

\begin{tabular}{llcccc}
\hline & & \multicolumn{2}{c}{ Positive Affection } & \multicolumn{2}{c}{ Negative Affection } \\
& & Mean difference & pbonf & Mean Difference & pbonf \\
\hline Baseline & Neutral & 0.337 & $0.011^{*}$ & 0.150 & 0.688 \\
& Positive Condition & 0.282 & $0.037^{*}$ & 0.179 & 0.332 \\
\hline
\end{tabular}

Int J Elec \& Comp Eng, Vol. 8, No. 5, October 2018 : 4004 - 4014 


\begin{tabular}{llcccc}
\hline & & \multicolumn{2}{c}{ Positive Affection } & \multicolumn{2}{c}{ Negative Affection } \\
& & Mean difference & pbonf & Mean Difference & pbonf \\
\hline \multirow{3}{*}{ Neutral Condition } & Negative Condition & 0.326 & $0.011^{*}$ & -0.168 & 0.848 \\
& Positive Condition & -0.055 & 1.000 & 0.029 & 1.000 \\
Positive condition & Negative Condition & -0.011 & 1.000 & -0.318 & $0.002^{* *}$ \\
& Negative Condition & 0.045 & 1.000 & -0.347 & $0.004 * *$ \\
\hline
\end{tabular}

In the PANAS sub-scale of positive affection, significant difference $(p<0.05)$ occurred in the baseline condition rather than in neutral condition $(p=0.011)$, positive condition $(p=0.037)$, and negative condition. It meant that providing the treatment had successfully changed the condition of the subject from the baseline condition based on the result of the PANAS scale. Descriptively, the condition of positive affection treatment showed higher mean (3.284) than that in the neutral condition $($ mean $=3.229)$ and negative condition (mean $=3.239)$. The difference was not statistically significant $(p>0.05)$, which meant that giving the treatment was inadequately effective in inducing the valence of different kinds of affection. On the PANAS sub-scale of negative affection, significant differences occurred in negative conditions rather than in positive condition $(\mathrm{p}=0.004 ; \mathrm{p}<0.01)$ and neutral condition $(\mathrm{p}=0.002 ; \mathrm{p}<0.01)$. It indicated that based on the PANAS scale, the provision of negative affection treatment had successfully induced negative affection, relative to the other two conditions. Negative treatment condition descriptively increased negative affection rather than the baseline condition (mean difference $=-0.222$ ) although it was not statistically significant. The researchers concluded that the provision of affection induction had successfully changed the positive affection condition from the baseline although it was not yet affective to differentiate it from other treatment condition. Meanwhile, the result of the negative affection sub-scale suggested that negative treatment condition could induce negative affection relative to other treatment conditions. There were possible measurement errors caused by the positive affection sub-scale of PANAS scale since some items had a relatively low inter-correlation among them. Furthermore, the process of classifying the GSR data to see the emotional status of the respondents will be discussed in the next chapter.

\subsection{Classification of GRS data}

The data resulted by the GSR were categorized into three, namely positive, neutral and negative according to the condition in time of data collection. The result of the data category was still in the form of time series. For the sake of SVM algorithm training, the data would be separated by class or cut by the category. Examples of the data categorization can be seen in Figure 4, Figure 5 and Figure 6.

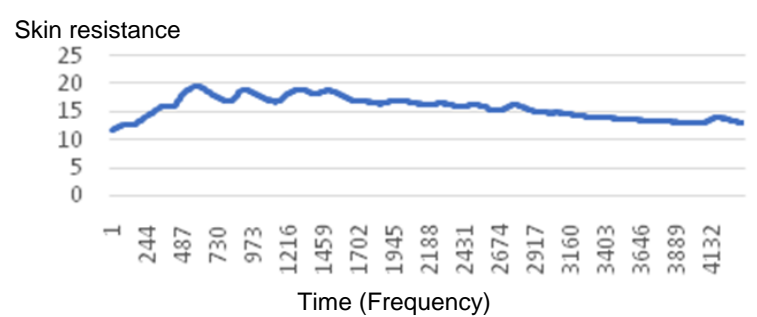

Figure 4. Example of positive data

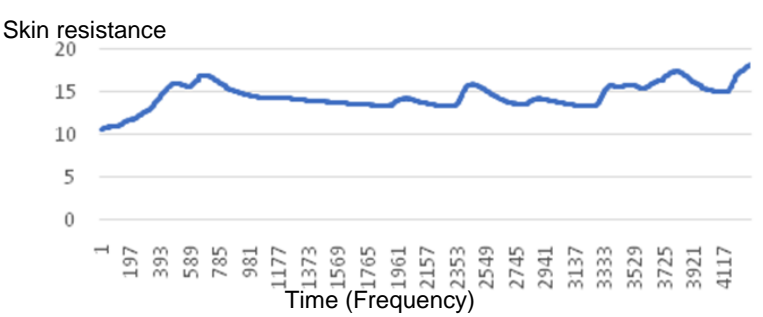

Figure 5. Example of neutral data

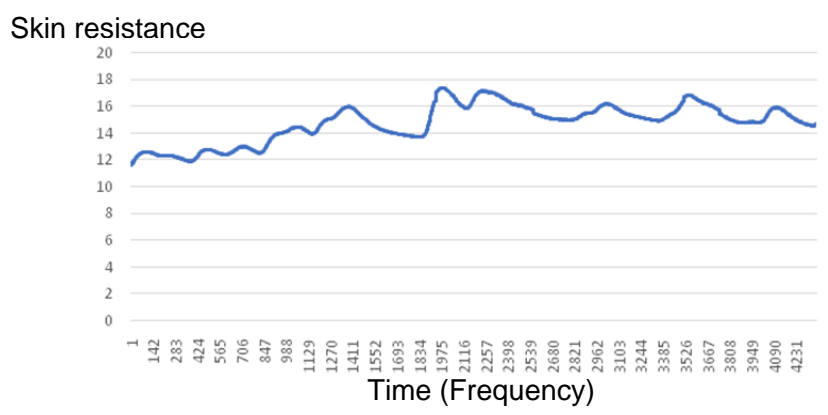

Figure 6. Example of negative data 


\subsection{Preprocessing}

There are three data preprocessing performed in this research, i.e., Aggregation, Normalization/Feature Scaling, Lagging, and Labelling. Data aggregation was done by changing the time unit from frequency into second. In data mining, aggregate data is useful and is aimed to increase the signal/noise ratio commonly, especially on the temporal data processing. The result of the data aggregation can be seen in Figure 7. The result showed that the data still have the same patterns with those before the aggregation. Normalization is a rescaling of the data from the original range so that all values are within the range of 0 and 1 .

Normalization can be useful and even required in some machine learning algorithms when your time series data has input values with differing scales. It may be needed for any classification algorithms which use distance as a foundation of data processing. The result of data normalization in the research can be seen in Figure 8. The last preprocessing is Lagging and Labelling. Lagging on data functions is preparing and framing the time series data. Example of lagging and labeling on data with five lags can be seen in Table 4 .

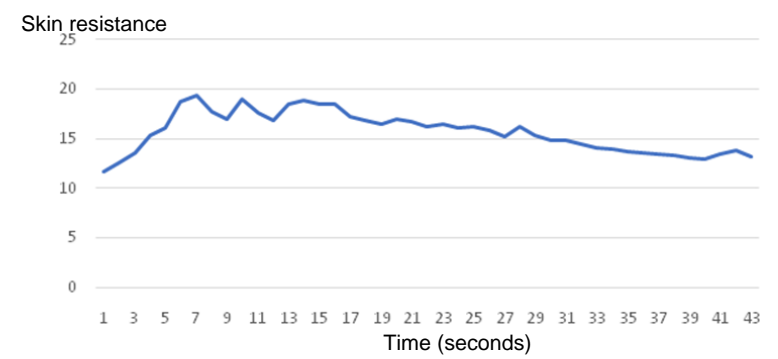

Figure 7. Example of positive aggregation

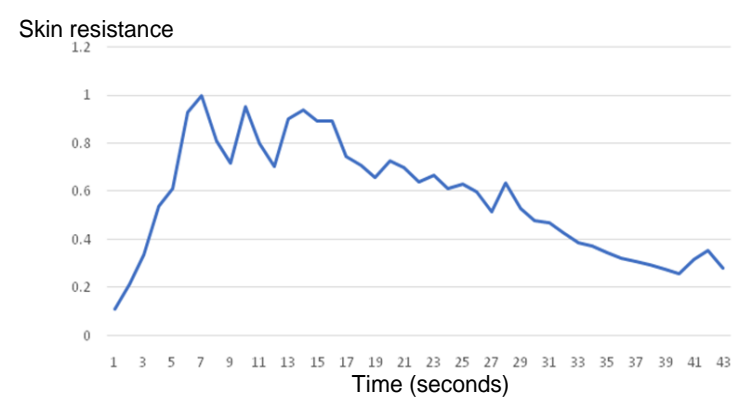

Figure 8. Example of normalization of positive data

Table 4. Example 5 rows of Data Lagging and Labeling

\begin{tabular}{cccccc}
\hline lag.1 & lag.2 & lag.3 & lag.4 & lag.5 & label \\
\hline 0.109827 & 0.216149 & 0.334738 & 0.539203 & 0.613395 & positive \\
0.216149 & 0.334738 & 0.539203 & 0.613395 & 0.929314 & positive \\
0.334738 & 0.539203 & 0.613395 & 0.929314 & 1 & positive \\
0.539203 & 0.613395 & 0.929314 & 1 & 0.812889 & positive \\
0.613395 & 0.929314 & 1 & 0.812889 & 0.717254 & positive \\
\hline
\end{tabular}

\subsection{Model and validation}

In this research, the processed data were classified with different kinds of lags and kernels to see the comparison of accuracy rate between the kernels and different numbers of lags. The data were classified by using a library "e1071" in language R. For the training, random sampling as much as $70 \%$ was used and the validation used the rest $30 \%$ of the data. The classification started with the 5 to 41 lags. The result of accuracy comparison in the classification model can be seen in Figure 9.

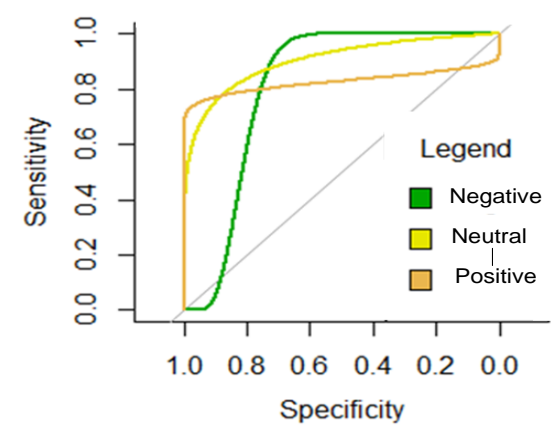

Figure 9. ROC graph 
It can be seen in Figure 9 that the accuracy level tends to increase along with the increasing number of lags. It is also noticed that the RBF kernels suggested the best performance compared to the kernels and algorithms of other classifications. A detailed result of this process is shown in Table 5. This table shows that SVM model using RBF kernels is the best, which was $75.65 \%$. The graph of ROC curve was visualized the classification result with the highest accuracy, which was $75.65 \%$, and it can be seen in Figure 9 . Figure 9 shows the mean of the AUC is 0.8019 . It indicated that the result of the emotion classification was quite good as the mean of the AUC was close to 1.

\begin{tabular}{ccccc}
\multicolumn{5}{c}{ Table 5. The Percentage Accuracy Comparison } \\
\hline Lag & RBF & Linear & Naïve Bayes & KNN \\
\hline 24 & 0.5220 & 0.4389 & 0.4627 & 0.4915 \\
25 & 0.5589 & 0.4285 & 0.4660 & 0.4875 \\
26 & 0.5932 & 0.4500 & 0.4858 & 0.5122 \\
27 & 0.6068 & 0.4371 & 0.4730 & 0.5329 \\
28 & 0.6157 & 0.4161 & 0.4288 & 0.5265 \\
29 & 0.5896 & 0.4285 & 0.4421 & 0.5328 \\
30 & 0.6262 & 0.4101 & 0.4927 & 0.5072 \\
31 & 0.6073 & 0.3795 & 0.4790 & 0.5418 \\
32 & 0.6080 & 0.4005 & 0.4375 & 0.5056 \\
33 & 0.6223 & 0.4055 & 0.4953 & 0.5294 \\
34 & 0.5939 & 0.4095 & 0.4368 & 0.5802 \\
35 & 0.7110 & 0.3878 & 0.4410 & 0.4790 \\
36 & 0.6538 & 0.3803 & 0.4529 & 0.5213 \\
37 & 0.6520 & 0.4362 & 0.4852 & 0.5343 \\
38 & 0.6322 & 0.2816 & 0.4310 & 0.4712 \\
39 & 0.7083 & 0.4583 & 0.4930 & 0.5069 \\
40 & 0.7565 & 0.4260 & 0.5478 & 0.5391 \\
41 & 0.7529 & 0.3411 & 0.4941 & 0.5764 \\
\hline
\end{tabular}

\section{DISCUSSION}

From the results obtained, it appeared that the higher the lag was, the higher the accuracy would tend to be in the RBF kernel and it was linear. It was known in the RBF kernel that to perform the classification of human emotional status, a large GSR pattern was required, which was 40 lags or 40 seconds (1 lag consists of 1-second data) so that the emotional status could be well classified. Similar results were also encountered by Grimes and Li in their previous studies. They obtained accuracy that tended to increase along with the increasing number of the lags that were used. However, Li experienced a decrease in accuracy and argued that the use of large lag would cause the computing process to become larger and not all datasets would benefit as the number of lags used [30], [31]. In his research, Widodo argued that determining the number of lag was an essential issue since too large lags could carry irrelevant information while too small lags might discard relevant information [32], [33]. It was also mentioned by Liu in her/his research. She/He said that the use of too large lags could disrupt the classification process because it made the data irrelevant [25]. Widodo's and Liu's opinion can be used to explain the incidence experienced by Li, where accuracy would increase along with increasing the number of lags but would decrease after passing a certain number of lags. This condition is different from the opinion given by Grimes and Solovey. They argued that lag was notable when the classification would be operated in real-time [30], [34]. Thus, the lag required to form the relevant data in classifying human emotional status was 40 since it could generate the highest accuracy in this classification, which was $75.65 \%$.

Normalization using feature scaling method also directly affected the results of classification. Classification without going through the feature scaling process could only achieve accuracy as much as $60.87 \%$, but with the use of scaling feature the accuracy could reach up to $15 \%$ increase, amounting to $75.65 \%$ because the process of scaling feature made the data on each respondent equivalent. Accordingly, the classification process was not disturbed by the data range that was too large or too small. This opinion was supported by Hsu who stated that performing feature scaling before classification using the SVM was essential as data with too large range could dominate data with a smaller range. Also, feature scaling process also facilitated the computation process [10]. Berg specifically conducted a study on comparing the impacts of various kinds of pre-processing on biological data. From the research, it could be concluded that the preprocessing had big implications on biological data, especially auto scaling and feature scaling. Prevoius result also said that the feature scaling process was related to biological data [35], [36]. Comparison of the impacts of normalization on the results of the classification of human emotional status can be seen in Table 6 . 
Table 6. Effect of Normalization to an Accuracy

\begin{tabular}{cc}
\hline Normalization & Accuracy \\
\hline With & 75.65 \\
Without & 60.87 \\
\hline
\end{tabular}

In addition to data processing, the use of the kernel on SVM also generated different results. In comparison, the RBF kernel could provide classification results with an accuracy of $75.65 \%$, while the results of accuracy by using other kernels such as linear, polynomial and sigmoid was under the accuracy level which the RBF kernel generated. Comparison of kernel usage to accuracy result of the classification can be seen in Table 7 . Classification result by using RBF was also validated by using $k$ Fold Cross Validation by using $k$ as much as 10. After doing ten times of validation using the method, the accuracy value obtained was $73 \%$ at the minimum and $78 \%$ at the maximum. The result showed that the data used in the classification process was not linear and the RBF kernel was able to generate the best accuracy in the classification of time series data using sliding windows technique. This result is in line with the opinion stated by Hsu, which said that the RBF kernel had often been used to classify nonlinear data in a bigger dimensional space [10].

Table 7. Comparison of Data Accuracy to different Kernels

\begin{tabular}{cc}
\hline Kernel & Accuracy \\
\hline RBF & 75.65 \\
Polynomial & 53.04 \\
Linear & 41.74 \\
Sigmoid & 28.7 \\
\hline
\end{tabular}

\section{CONCLUSION}

We have developed a model of a system for detecting emotion from galvanic skin respond data. Our research aimed to develop a model which can use sensor information to detect the emotion status of the respondents. PANAS scale is applied to validate the data set for the training process. Once verified, a dataset is used for training SVM. The experiments on emotion determination from audio-visual induction to emotional detection showed the performance of the system. The result indicates that the model is well performed since the accuracy is about $75.65 \%$ after some pre-processing is applied. More exploration is also required to determine the lag of the data due to the variability in the respondents.

\section{ACKNOWLEDGMENTS}

I would like to thank Universitas Atma Jaya Yogyakarta, Indonesia for the financial support to our research project. I would also like to express my gratitude to all members of Cognitive and Affective Neuroscience Research Group, Psychology Department, Gadjah Mada University for providing time to conduct an experimental process of our research.

\section{REFERENCES}

[1] N. Sebe, et al., "Multimodal Emotion Recognition", Handbook of Pattern Recognition and Computer Vision, vol. 4, pp. 387-419, 2005.

[2] B. Meuleman and K. R. Scherer, "Onlinear appraisal modeling: An application of machine learning to the study of emotion production", IEEE Transactions on Affective Computing, vol. 4, no. 4, pp. 398-411, 2013.

[3] E. H. Jang, et al., "Emotion Classification based on Bio-signals Emotion Recognition using Machine Learning Algorithms", vol. 3, pp. 1373-1376, 2014.

[4] K. Ansari-Asl, et al., "Channel Selection Method for Eeg Classification in Emotion Assessment based on Synchronization Likelihood", European Signal Processing Conference (EUSIPCO), 2007.

[5] Y. Liu, et al., "Real-time EEG-based Human Emotion Recognition and Visualization", CW'10 Proceedings of the 2010 International Conference on Cyberworlds, pp. 262-269, 2010.

[6] F. Ringeval, et al., "Prediction of Asynchronous Dimensional Emotion Ratings from Audiovisual and Physiological Data", Pattern Recognition Letters, vol. 66, pp. 22-30, 2015.

[7] X. Wu, et al., "Top 10 algorithms in data mining", Knowledge and information systems, vol. 14, no. 1, pp. 1-37, 2008.

[8] H. Y. Huang and C. J. Lin, "Linear and Kernel Classification: When to use which?", Proceedings of the 2016 SIAM International Conference on Data Mining, Society for Industrial and Applied Mathematic, pp. 216-224, 2016.

[9] C. Cortes and V. Vapnik, "Support-Vector Networks, Machine Learning", Kluwer Academic Publishers, Boston, pp. 273-297, 1995. 
[10] C. W. Hsu, et al., "A Practical Guide to Support Vector Classification”, 2016.

[11] Shimmer, "Measuring Emotion: Reactions to Media", Dublin, Ireland, 2015.

[12] M. Liu, et al., "Human Emotion Recognition based on Galvanic Skin Response Signal Feature Selection and SVM", Smart City and Systems Engineering (ICSCSE), International Conference on, pp. 157-160, 2016.

[13] K. H. Kim, et al.., "Emotion Recognition System using short-term Monitoring of Physiological Signals", Medical and Biological Engineering and Computing, vol. 42, no. 3, pp. 419-427, 2004.

[14] H. Kurniawan, et al., "Stress Detection from Speech and Galvanic skin response Signals", Computer-Based Medical Systems (CBMS), IEEE 26th International Symposium on, pp. 209-214, 2013.

[15] N. Nourbakhsh, et al., "Using Galvanic Skin Response for Cognitive Load Measurement in Arithmetic and Reading Tasks", 24th Australian Computer Human Interaction Conference, pp. 420-423, 2012.

[16] P. Shangguan, et al., "The Emotion Recognition Based on GSR Signal by Curve Fitting", J. Information and Computational Science, vol. 11, no. 8, pp. 2635-2646, 2014.

[17] C. Setz, et al., "Discriminating Stress from Cognitive load using a Wearable EDA Device", IEEE Transactions on Information Technology in Biomedicine, vol. 14, no. 2, pp. 410-417, 2010.

[18] R. Guo, et al., "Pervasive and Unobtrusive Emotion Sensing for Human Mental Health", Pervasive Health '13 Proceedings of the 7th International Conference on Pervasive Computing Technologies for Healthcare, pp. 436-439, 2013.

[19] A. Nakasone, et al., "Emotion Recognition from Electromyography and Skin Conductance", Proc. of the 5th International Workshop on Biosignal Interpretation, pp. 219-222, 2005.

[20] M. Stikic, et al., "EEG-based Classification of Positive and Negative Affective States", Brain-Computer Interfaces, vol. 1, no. 2, pp. 99-112, 2014.

[21] Y. Lu, et al., "Combining Eye Movements and EEG to Enhance Emotion Recognition", Proceedings of the TwentyFourth International Joint Conference on Artificial Intelligence (IJCAI), 2015.

[22] M. V. Villarejo, et al., "A Stress Sensor based on Galvanic Skin Response (GSR) Controlled by ZigBee", Sensors, vol. 12, no. 5, pp. 6075-6101, 2012.

[23] K. Khalfallah, et al., "Non invasive Galvanic Skin Sensor for Early Diagnosis of Sudomotor Dysfunction: Application to Diabetes", IEEE Sensor J., vol. 12, pp. 456-463, 2010.

[24] K. Setz, et al., "Towards Long Term Monitoring of Electrodermal Activity in Daily Life", Proceedings of 5th International Workshop on Ubiquitous Health and Wellness, Copenhagen, Denmark, 2010.

[25] D. Liu and M. Ulrich, "Listen to Your Heart: Stress Prediction using Consumer Heart Rate Sensors", CS229 Machine Learning, Autumn 2013.

[26] V. N. Vapnik, "The Nature of Statistical Learning Theory”, Springer-Verlag New York, Inc., 1995.

[27] C. Campbell and Y. Ying, "Learning with Support Vector Machines", Morgan \& Claypool Publishers, 2011.

[28] J. R. Crawford and J. D. Henry, "The Positive and Negative Affect Schedule (PANAS): Construct validity, measurement properties and normative data in a large non-clinical sample", British Journal of Clinical Psychology, vol. 43, no. 3, pp. 245-265, 2004.

[29] S. B. Gunawan, "Deteksi Status Emosi Manusia Dengan Algoritma Support Vector Machine Menggunakan Data Galvanic Skin Response", Theses, Universitas Atma Jaya Yogyakarta, 2017.

[30] D. Grimes, et al., "Feasibility and Pragmatics of Classifying", CHI 2008 Proceedings: Cognition, Perception, and Memory, 2008.

[31] X. Li and B. Marlin, "Classification of Sparse and Irregularly Sampled Time Series with”, Proceeding UAI'15 Proceedings of the Thirty-First Conference on Uncertainty in Artificial Intelligence, pp. 484-493, 2015.

[32] I. B. Widodo and B. Widjaja, "Automatic Lag Selection in time series Forecasting using Multiple Kernel Learning", International Journal of Machine Learning and Cybernetics, vol. 7, no. 1, pp. 95-110, 2016.

[33] E. T. Solovey, et al., "Classifying Driver Workload using Physiological and Driving Performance Data: Two Field Studies", Proceeding CHI '14 Proceedings of the SIGCHI Conference on Human Factors in Computing Systems, pp. 4057-4066, 2014

[34] A. Ambarwari, et al., "Biometric Analysis of Leaf Venation Density Based on Digital Image", Telecommunication Computing Electronics and Control, vol. 16, no. 4, 2018.

[35] R. A. v. d. Berg, et al., "Centering, Scaling, and Transformations: Improving the Biological Information Content of Metabolomics Data", BMC Genomics, 2006.

[36] K. Daimi and S. Banitaan, "Using Data Mining to Predict Possible future Depression cases", International Journal of Public Health Science (IJPHS), vol. 3, no. 4, pp. 231-240, 2014.

\section{BIOGRAPHIES OF AUTHORS}

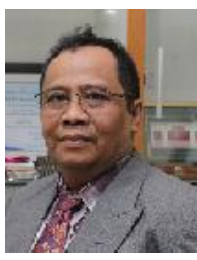

Djoko Budiyanto Setyohadi received the B.E. degree in Electrical Engineering from Universitas Gadjah Mada, Yogyakarta, Indonesia in 1990, the M.Eng. degree in Computer Science, Information Management from the Asian Institute of Technology, Bangkok, Thailand, in 1998 and the Ph.D. degree in Computer Science from University Kebangsaan Malaysia. He is an Associate Professor of Informatics Engineering at the Faculty of Industrial Engineering, Universitas Atma Jaya Yogyakarta. His current research interests include Information System, Human Computer Interface and Data Engineering. 


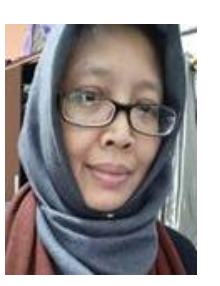

Sri Kusrohmaniah received the B.E. degree in Psychology from UniversitasGadjahMada, Yogyakarta, Indonesia while her Ph.D. degree in Psychology from University Kebangsaan Malaysia, in 2017. She is a senior lecturer in Psychology Department, Gadjah Mada University Yogyakarta. Her current research interests include Neuroscience, Human Computer Interface and Experimental Psychology.

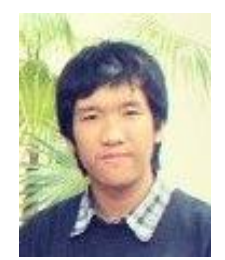

Sebastian Bagya Gunawan received the Bachelor Degree in 2017 from University of Atma Jaya Yogyakarta majoring in Information System. He is a research assistant in Data Engineering and InformationSystem Research Group. His current research interests include Human Computer Interface and Data Engineering.

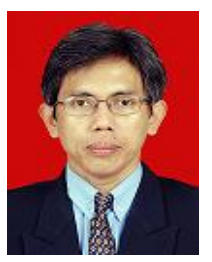

Pranowo received the B.Eng. Degree in Mechanical Engineering from UniversitasGadjahMada, Indonesia, in 1996, the M.Eng. degree in electrical engineering from the Universitas Gadjah Mada, Indonesia, in 2002 and Ph.D. degree in Electrical Engineering and Information Technology from Universitas Gadjah Mada, Indonesia, in 2010. He is currently a Senior Lecturer in Department of InformaticEngineering with the Faculty of Industrial Technology, Universitas Atma Jaya Yogyakarta, Indonesia. His research interests include numerical simulation and modeling, computer vision and parallel programming.

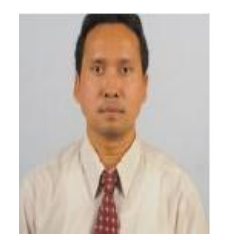

Anton Satria Prabuwono is currently a Professor in Faculty of Computing and Information Technology in Rabigh, King Abdulaziz University, Saudi Arabia. He joined Faculty of Information Science and Technology, University Kebangsaan Malaysia (UKM) in 2009. He then joined Faculty of Computing and Information Technology in Rabigh, King Abdulaziz University in 2013. He served as Editor, Technical Committee, and Reviewer in many International Journals and Conferences. He is a senior member of IEEE and a member of ACM. In general, his research interests include computer vision, intelligent robotics, and autonomous systems. 